\title{
Rhodium-catalyzed synthesis of C4-chalcogenoalkylated oxindoles via Sommelet-Hauser type rearrangement of 3-diazoindolin-2-ones
}

\author{
ANGULA CHANDRA SHEKAR REDDY, BHOJKUMAR NAYAK and \\ PAZHAMALAI ANBARASAN* (D) \\ Department of Chemistry, Indian Institute of Technology Madras, Chennai 600 036, Tamilnadu, India \\ E-mail: anbarasansp@iitm.ac.in
}

MS received 30 July 2019; revised 31 October 2019; accepted 4 November 2019; published online 3 December 2019

\begin{abstract}
Efficient rhodium-catalyzed Sommelet-Hauser type rearrangement of 3-diazoindolin-2-ones with $\alpha$-thioesters has been accomplished for the synthesis of $\mathrm{C} 4$-thioalkylated oxindoles. The developed reaction offers the selective functionalization of $\mathrm{C} 4$-position of oxindole via generation of S-ylide and [2, 3]-sigmatropic rearrangement and allows access to diverse $\mathrm{C} 4$-thioalkylated oxindoles in good to excellent yield. Furthermore, the method was successfully extended to the synthesis $\mathrm{C} 4$-selenoalkylated oxindoles employing the corresponding $\alpha$-selenoester.
\end{abstract}

Keywords. $\alpha$-Thioesters; $\alpha$-selenoesters; 3-diazoindolin-2-ones; S-ylide; Se-ylide; oxindoles; SommeletHauser rearrangement.

\section{Introduction}

Substituted oxindoles have drawn special attention in synthetic organic and medicinal chemistry due to their widespread biological activity. ${ }^{1}$ For instance, the drug molecule having oxindoles skeletons exhibits extensive bio-activities, ${ }^{2}$ which include anti-HIV, protein kinase inhibitors, ${ }^{3}$ anti-bacterial agents, etc. ${ }^{4}$ Some of the representative examples of therapeutically important oxindole containing molecules are shown in Figure 1. In addition, aryl sulfonanilide substituted oxindole hybrid also exhibits enhanced activity and inhibits the growth of cancer cells by partial depletion of intracellular calcium stores and phosphorylation of elF $2 \alpha .^{5}$ Similarly, C4-substituted oxindole derivatives also show diverse biological activities. ${ }^{6}$ Due to their biological importance, during past decades, numerous methods for the synthesis of oxindoles have been developed. ${ }^{7}$ Most of the known methods are limited to the construction of pyrrolidone ring system of oxindole from functionalized arene and selective functionalization of oxindole is rather limited. ${ }^{8}$ Hence, the development of a practical and general method for the selective synthesis of substituted oxindole, which will minimize the number of steps and increases complexity and diversity, is an attractive subject in organic synthesis. ${ }^{9}$

On the other hand, diazocarbonyl compounds, a vital coupling partner in organic synthesis for the construction of various complex molecule, ${ }^{10}$ in the presence of metal catalyst generates important reactive intermediate, viz, metal carbeniods. This intermediate undergoes various useful transformations including insertion into $\mathrm{C}-\mathrm{H}^{11}$ and $\mathrm{X}-\mathrm{H} / \mathrm{C}$ bond, ${ }^{12}$ ylide generation-cum-functionalization, ${ }^{13}$ etc. Among these transformations, ylide generation from diazo compound and Lewis base, in particular, sulfides, ${ }^{14}$ followed by either $[2,3] /[1,2]$-sigmatrophic rearrangement or Sommelet-Hauser type rearrangement ${ }^{15}$ affords an interesting possibility for the construction of diverse sulfur-based building blocks and heterocycles. ${ }^{16}$ Although $[2,3] /[1,2]$-sigmatrophic rearrangements of sulfur ylides have been extensively studied, Sommelet-Hauser type rearrangement of sulfur ylides is rather limited. For instance, rhodium-catalyzed Sommlet-Hauser type rearrangement of sulfur ylide was disclosed by Wang and coworkers, ${ }^{17}$ wherein the reaction of aryl-substituted diazoacetates with ethyl phenylthioacetate in the presence of $\mathrm{Rh}_{2}\left(\mathrm{O}_{2} \mathrm{CCF}_{3}\right)_{4}$ affords ortho-substituted arylacetates

*For correspondence

Electronic supplementary material: The online version of this article (https://doi.org/10.1007/s12039-019-1711-9) contains supplementary material, which is available to authorized users. 
<smiles>[R2]C(=O)C1CC12C(=O)Nc1ccccc12</smiles>

HIV-1 inhibitors<smiles>CC(C)c1ccc(CCN)c2c1N(C)C(=O)C2</smiles>

Dopamine

Receptor agonist<smiles>CC1(C)C(=O)Nc2ccc(C3=NNC(=O)CC3)cc21</smiles>

Indolidan<smiles>Cc1cc(C2(c3ccccc3)C(=O)Nc3ccccc32)c(NS(=O)(=O)c2ccc(C(C)(C)C)cc2)cc1O</smiles>

Arylsulfonanilide-oxindole hybrid

Figure 1. Biologically important oxindole derivatives.

(Scheme 1a). Inspired from this work and our continued interest in functionalization of metal carbenoids, ${ }^{18}$ we envisioned the rhodium-catalyzed Sommelet-Hauser type rearrangement of sulfur ylides derived from 3-diazoindolin-2-ones and $\alpha$-thioester (Scheme 1b). The successful development of the reaction would offer selective rhodium-catalyzed synthesis of C4-thioalkylated oxindole, a biologically important structural motif.

\section{Experimental}

\subsection{General information}

All reactions were carried out under an atmosphere of dry nitrogen using reaction tubes. Dry dichloromethane (DCM) was prepared by distilling over calcium hydride and stored over $4 \AA$ molecular sieves under $\mathrm{N}_{2}$ atmosphere. All the 3-diazoindolin-2ones were synthesized from isatin and tosylhydrazine employing literature procedure. ${ }^{19} \alpha$-Thioester ${ }^{20}$ and $\alpha$-selenoester ${ }^{21}$ were prepared using literature protocol. Column chromatography was performed using Rankem Silicagel (100-200 mesh) and the solvent system used unless otherwise specified, was ethyl acetate-hexanes with various percentage of polarity depending on the nature of the substrate. NMR data were recorded on Bruker DPX $400 \mathrm{MHz}$ spectrometers. ${ }^{13} \mathrm{C}$ and ${ }^{1} \mathrm{H}$ NMR spectra were referenced to signals of deutero and residual protiated solvents, respectively. Infrared spectra were recorded on a Thermo Nicolet iS10 FT spectrometer. HRMS were

(a) Known work

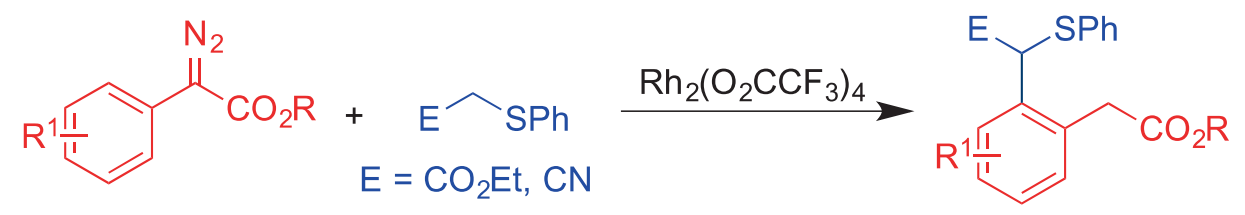

(b) This work

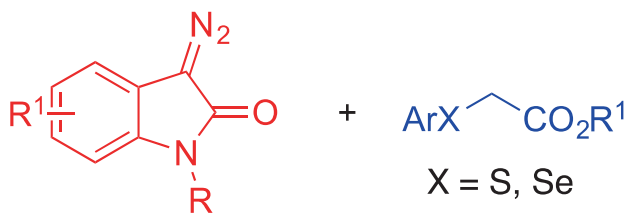

[Rh]<smiles>[R]OC(=O)C([Y10])C1=[R1]#CC2=C1CC(=O)N2[R]</smiles>

C4-substituted oxindole

Scheme 1. Rhodium catalyzed Sommelet-Hauser type rearrangement. 
recorded by electron spry ionization (ESI) method on a Q-TOF Micro with lock spray source.

\subsection{Typical procedure for the rhodium-catalyzed synthesis of substituted oxindole 3}

$\alpha$-Thioester/ $\alpha$-selenoester 1 (0.4 mmol, 1 equiv), $\mathrm{Rh}_{2}(\mathrm{OAc})_{4}(0.008 \mathrm{mmol}, 2 \mathrm{~mol} \%)$ and dichloromethane $(2 \mathrm{~mL})$ were added under nitrogen atmosphere to an oven-dried $10 \mathrm{~mL}$ reaction tube equipped with stir bar. The reaction tube was sealed with septa and stirred at room temperature. Subsequently, the solution of 3-diazoindolin-2-one 2 ( $0.8 \mathrm{mmol}, 2$ equiv) in dichloromethane $(0.3 \mathrm{~mL})$ was introduced slowly through syringe pump (addition rate $=0.01 \mathrm{~mL} / \mathrm{min}$ ). After the addition of diazo compound, the reaction mixture was stirred for $2 \mathrm{~h}$ at same temperature. After the TLC analysis, solvent was removed under reduced pressure. Purification of the resultant crude through column chromatography using mixture of hexane/ethyl acetate as an eluent afforded oxindole $\mathbf{3}$ in high yield and purity

3a: Brick red solid; yield: $81 \%$; M.p.: $98-100{ }^{\circ} \mathrm{C}$; $\mathrm{R}_{f}$ $=0.55$ in 30:70 ethyl acetate/hexane; FTIR (KBr): 3061, 2931, 2362, 1719, 1606, 1469, 1348, 1301, 1253, 1150, 1095, 1026, $982 \mathrm{~cm}^{-1}$; ${ }^{1} \mathrm{H}$ NMR (400 $\left.\mathrm{MHz}, \mathrm{CDCl}_{3}\right): \delta 7.38-7.32(\mathrm{~m}, 2 \mathrm{H}), 7.30-7.15(\mathrm{~m}$, $5 \mathrm{H}), 6.75(\mathrm{~d}, 1 \mathrm{H}, J=7.6 \mathrm{~Hz}), 4.85(\mathrm{~s}, 1 \mathrm{H}), 4.24-4.03$ $(\mathrm{m}, 2 \mathrm{H}), 3.59$ and $3.34(\mathrm{ABq}, 2 \mathrm{H}, J=22.1 \mathrm{~Hz}), 3.18$ $(\mathrm{s}, 3 \mathrm{H}), 1.18(\mathrm{t}, 3 \mathrm{H}, J=7.1 \mathrm{~Hz}) ;{ }^{13} \mathrm{C}\left\{{ }^{1} \mathrm{H}\right\} \mathrm{NMR}(100$ $\left.\mathrm{MHz}, \mathrm{CDCl}_{3}\right): \delta 174.6,169.6,145.3,133.2,133.1$, 132.1, 129.1, 128.5, 128.4, 123.7, 122.2, 107.9, 62.0, 54.4, 34.7, 26.3, 14.0; HRMS: calcd. for $\mathrm{C}_{19} \mathrm{H}_{19} \mathrm{NO}_{3} \mathrm{~S}$ $+\mathrm{Na}$ : 364.0978; found: 364.0982 .

3b: Red semi solid; yield: $76 \%$; $\mathrm{R}_{f}=0.39$ in $30: 70$ ethyl acetate/hexane; FTIR (KBr): 3056, 2982, 2850, 2306, 1717, 1605, 1469, 1352, 1262, 1156, 1102, 1030, $900 \mathrm{~cm}^{-1} ;{ }^{1} \mathrm{H}$ NMR (400 $\left.\mathrm{MHz}, \mathrm{CDCl}_{3}\right): \delta$ 7.32-7.29 (m, 1H), 7.8-7.23 (m, 2H), $7.16(\mathrm{~d}, 1 \mathrm{H}, J=$ $7.9 \mathrm{~Hz}), 6.82-6.77(\mathrm{~m}, 1 \mathrm{H}), 6.77-6.70(\mathrm{~m}, 2 \mathrm{H}), 4.71$ (s, 1H), 4.24-4.01 (m, 2H), $3.77(\mathrm{~s}, 3 \mathrm{H}), 3.56$ and 3.32 $(\mathrm{ABq}, 2 \mathrm{H}, J=21.8 \mathrm{~Hz}), 3.18(\mathrm{~s}, 3 \mathrm{H}), 1.19$ (t, 3H, $J=$ $7.0 \mathrm{~Hz}) ;{ }^{13} \mathrm{C}\left\{{ }^{1} \mathrm{H}\right\}$ NMR $\left(100 \mathrm{MHz}, \mathrm{CDCl}_{3}\right): \delta 174.6$, $169.7,160.4,145.3,136.4,132.3,128.4,123.7,123.2$, 122.2, 114.6, 107.8, 61.9, 55.3(9), 55.3(3), 34.7, 26.3, 14.1; HRMS: calcd. for $\mathrm{C}_{20} \mathrm{H}_{21} \mathrm{NO}_{4} \mathrm{~S}+\mathrm{H}$ : 372.1264 ; found: 372.1279 .

3c: Red semi solid; yield: $64 \% ; \mathrm{R}_{f}=0.47$ in $30: 70$ ethyl acetate/hexane; FTIR (KBr): 3055, 2980, 2931, 2372, 2307, 1718, 1608, 1468, 1351, 1298, 1262, 1149, 1098, 1031, $738 \mathrm{~cm}^{-1}$; ${ }^{1} \mathrm{H}$ NMR (400 MHz, $\left.\mathrm{CDCl}_{3}\right): \delta 7.31-7.18(\mathrm{~m}, 3 \mathrm{H}), 7.00(\mathrm{~s}, 1 \mathrm{H}), 6.89(\mathrm{~d}$,
$1 \mathrm{H}, J=7.7 \mathrm{~Hz}), 6.74(\mathrm{~d}, 1 \mathrm{H}, J=7.1 \mathrm{~Hz}), 4.71(\mathrm{~s}, 1 \mathrm{H})$, $4.20-4.02(\mathrm{~m}, 2 \mathrm{H}), 3.58$ and $3.29(\mathrm{ABq}, 2 \mathrm{H}, J=21.5$ $\mathrm{Hz}), 3.18$ (s, 3H), 2.34 (s, 3H), 2.27 (s, 3H), 1.17 (t, $3 \mathrm{H}, J=7.1 \mathrm{~Hz}) ;{ }^{13} \mathrm{C}\left\{{ }^{1} \mathrm{H}\right\} \mathrm{NMR}\left(100 \mathrm{MHz}, \mathrm{CDCl}_{3}\right): \delta$ 174.6, 169.8, 145.3, 141.2, 139.0, 134.6, 132.3, 131.4, $128.7,128.5,127.4,123.7,122.3,107.8,61.9,53.8$, 34.7, 26.3, 21.1, 20.6, 14.0; HRMS: calcd. for $\mathrm{C}_{21} \mathrm{H}_{23} \mathrm{NO}_{3} \mathrm{~S}+\mathrm{Na}$ : 392.1291; found: 392.1298 .

3d: Red semi solid; yield: $71 \% ; \mathrm{R}_{f}=0.34$ in 40:60 ethyl acetate/hexane; FTIR (KBr): 3057, 2982, 2928, 2857, 2686, 2522, 2413, 2372, 2306, 2134, 1715, $1610,1504,1463,1422,1264,1179,1147,1096$, 1033, $740 \mathrm{~cm}^{-1}$; ${ }^{1} \mathrm{H}$ NMR (400 MHz, $\left.\mathrm{CDCl}_{3}\right): \delta$ 7.30-7.23 (m, 1H), $7.16(\mathrm{~d}, 1 \mathrm{H}, J=7.9 \mathrm{~Hz}), 7.04-6.96$ $(\mathrm{dd}, 1 \mathrm{H}, J=1.5,8.2 \mathrm{~Hz}), 6.79(\mathrm{~d}, 1 \mathrm{H}, J=1.5 \mathrm{~Hz}), 6.76$ (s, 1H), 6.74 (s, 1H), 4.75 (s, 1H), 4.21-4.09 (m, 2H), $3.85(\mathrm{~s}, 3 \mathrm{H}), 3.74(\mathrm{~s}, 3 \mathrm{H}), 3.58$ and $3.32(\mathrm{ABq}, 2 \mathrm{H}, J=$ $22.2 \mathrm{~Hz}), 3.19(\mathrm{~s}, 3 \mathrm{H}), 1.20(\mathrm{t}, 3 \mathrm{H}, J=7.0 \mathrm{~Hz})$; ${ }^{13} \mathrm{C}\left\{{ }^{1} \mathrm{H}\right\}$ NMR $\left(100 \mathrm{MHz}, \mathrm{CDCl}_{3}\right): \delta 174.5,169.7$, 150.0, 148.8, 145.3, 132.3, 128.8, 127.8, 123.8, 123.4, 122.3, 117.5, 111.3, 107.8, 62.0, 55.9(6), 55.9(2), 55.3, 34.7, 26.3, 14.1; HRMS: calcd. for $\mathrm{C}_{21} \mathrm{H}_{23} \mathrm{NO}_{5} \mathrm{~S}+$ Na: 424.1189; found: 424.1195 .

3e: Brick red liquid; yield: $78 \%$; $\mathrm{R}_{f}=0.49$ in 30:70 ethyl acetate/hexane; FTIR (KBr): 3057, 2982, 2928, $2857,2686,2522,2413,2372,2306,2134,1715$, $1610,1504,1463,1422,1264,1179,1147,1096$, 1033, $740 \mathrm{~cm}^{-1}$; ${ }^{1} \mathrm{H}$ NMR (400 $\left.\mathrm{MHz}, \mathrm{CDCl}_{3}\right): \delta$ 7.46-7.32 (m, 2H), 7.31-7.18 (m, 3H), $7.14(\mathrm{t}, 1 \mathrm{H}, J=$ $7.4 \mathrm{~Hz}), 6.75(\mathrm{~d}, 1 \mathrm{H}, J=7.4 \mathrm{~Hz}), 5.05(\mathrm{~s}, 1 \mathrm{H})$, $4.22-4.04(\mathrm{~m}, 2 \mathrm{H}), 3.67$ and $3.49(\mathrm{ABq}, 2 \mathrm{H}, J=22.1$ $\mathrm{Hz}), 3.19$ (s, 3H), $1.16(\mathrm{t}, 3 \mathrm{H}, J=6.9 \mathrm{~Hz}) ;{ }^{13} \mathrm{C}\left\{{ }^{1} \mathrm{H}\right\}$ NMR $\left(100 \mathrm{MHz}, \mathrm{CDCl}_{3}\right): \delta$ 174.6, 169.3, 145.5, $137.5,134.7,132.2,131.4,130.1,129.7,128.7,127.3$, $123.9,122.3,108.1,62.2,52.3,34.8,26.4,14.1$; HRMS: calcd. for $\mathrm{C}_{19} \mathrm{H}_{18} \mathrm{ClNO}_{3} \mathrm{~S}+\mathrm{Na}$ : 398.0588; found: 398.0597 .

3f: Orange liquid; yield: $75 \% ; \mathrm{R}_{f}=0.50$ in $30: 70$ ethyl acetate/hexane; FTIR (KBr): 3061, 2977, 2930, 2373, 2181, 2129, 1717, 1656, 1610, 1470, 1354, 1301, 1262, 1225, 1155, 1092, 1029, $817 \mathrm{~cm}^{-1} ;{ }^{1} \mathrm{H}$ NMR $\left(400 \mathrm{MHz}, \mathrm{CDCl}_{3}\right): \delta 7.36(\mathrm{t}, 1 \mathrm{H}, J=7.4 \mathrm{~Hz})$, 7.33-7.24 (m, 2H), $7.18(\mathrm{~d}, 1 \mathrm{H}, J=7.7 \mathrm{~Hz}), 7.09-7.00$ $(\mathrm{m}, 2 \mathrm{H}), 6.78(\mathrm{~d}, 1 \mathrm{H}, J=7.5 \mathrm{~Hz}), 4.99(\mathrm{~s}, 1 \mathrm{H})$, 4.23-4.01 (m, 2H), 3.65 and $3.48(\mathrm{ABq}, 2 \mathrm{H}, J=22.3$ $\mathrm{Hz}), 3.19$ (s, 3H), 1.15 (t, 3H, $J=7.1 \mathrm{~Hz}) ;{ }^{13} \mathrm{C}\left\{{ }^{1} \mathrm{H}\right\}$ NMR $\left(100 \mathrm{MHz}, \mathrm{CDCl}_{3}\right): \delta 174.6,169.5,145.5$, $136.0,131.7,131.4(\mathrm{~d}, J=51.2 \mathrm{~Hz}), 131.1,128.6$, $124.6(\mathrm{~d}, J=3.9 \mathrm{~Hz}), 123.9,122.2,116.1(\mathrm{~d}, J=22.2$ Hz), 108.1, 62.1, 52.6, 34.7, 26.4, 14.1; HRMS: calcd. for $\mathrm{C}_{19} \mathrm{H}_{18} \mathrm{FNO}_{3} \mathrm{~S}+\mathrm{Na}$ : 382.0884; found: 382.0888 .

3g: Red solid; yield: $80 \%$; M.p.: $110-112{ }^{\circ} \mathrm{C}$; $\mathrm{R}_{f}=$ 0.49 in 30:70 ethyl acetate/hexane; FTIR (KBr): 3057, 
2978, 2937, 2364, 2314, 1719, 1607, 1470, 1350, 1300, 1261, 1152, 1096, 1023, $983 \mathrm{~cm}^{-1} ;{ }^{1} \mathrm{H}$ NMR $\left(400 \mathrm{MHz}, \mathrm{CDCl}_{3}\right): \delta 7.31-7.18(\mathrm{~m}, 5 \mathrm{H}), 7.13(\mathrm{~d}, 1 \mathrm{H}$, $J=8.0 \mathrm{~Hz}), 6.76(\mathrm{~d}, 1 \mathrm{H}, J=7.6 \mathrm{~Hz}), 4.83(\mathrm{~s}, 1 \mathrm{H})$, $4.22-4.05(\mathrm{~m}, 2 \mathrm{H}), 3.63$ and $3.44(\mathrm{ABq}, 2 \mathrm{H}, J=22.5$ $\mathrm{Hz}), 3.19$ (s, 3H), 1.19 (t, 3H, $J=7.1 \mathrm{~Hz}) ;{ }^{13} \mathrm{C}\left\{{ }^{1} \mathrm{H}\right\}$ NMR $\left(100 \mathrm{MHz}, \mathrm{CDCl}_{3}\right): \delta 174.4,169.3,145.5$, $134.7,134.5,131.6(6), 131.6(0), 129.2,128.6,123.6$, 122.2, 108.1, 62.1, 54.4, 34.7, 26.3, 14.0; HRMS: calcd. for $\mathrm{C}_{19} \mathrm{H}_{18} \mathrm{ClNO}_{3} \mathrm{~S}+\mathrm{Na}$ : 398.0588; found: 398.0589 .

3h: Orange solid; yield: $81 \%$; M.p.: $154-156{ }^{\circ} \mathrm{C}$; $\mathrm{R}_{f}$ $=0.45$ in 30:70 ethyl acetate/hexane; FTIR (KBr): 3057, 2983, 2833, 2359, 2309, 1720, 1602, 1463, 1346, 1266, 1153, 1101, 1026, 944, $896 \mathrm{~cm}^{-1} ;{ }^{1} \mathrm{H}$ NMR $\left(400 \mathrm{MHz}, \mathrm{CDCl}_{3}\right): \delta 7.10-7.03(\mathrm{~m}, 3 \mathrm{H}), 6.98$ $(\mathrm{d}, 1 \mathrm{H}, J=7.9 \mathrm{~Hz}), 6.59-6.49(\mathrm{~m}, 3 \mathrm{H}), 4.56(\mathrm{~s}, 1 \mathrm{H})$, $4.07-3.83(\mathrm{~m}, 2 \mathrm{H}), 3.40$ and $3.16(\mathrm{ABq}, 2 \mathrm{H}, J=21.6$ $\mathrm{Hz}), 3.01(\mathrm{~s}, 3 \mathrm{H}), 1.01(\mathrm{t}, 3 \mathrm{H}, J=7.1 \mathrm{~Hz}), 0.78(\mathrm{~s}, 9 \mathrm{H})$, $0.01(\mathrm{~s}, 6 \mathrm{H}) ;{ }^{13} \mathrm{C}\left\{{ }^{1} \mathrm{H}\right\}$ NMR $\left(100 \mathrm{MHz}, \mathrm{CDCl}_{3}\right): \delta$ $174.5,169.7,156.7,145.3,136.3,132.3,128.4,124.1$, 123.8, 122.3, 120.8, 107.7, 61.9, 55.1, 34.7, 26.3, 25.6, 18.2, 14.1, -4.4; HRMS: calcd. for $\mathrm{C}_{25} \mathrm{H}_{33} \mathrm{NO}_{4} \mathrm{SSi}+$ Na: 494.1792; found: 494.1789.

3i: Red solid; yield: $88 \%$; M.p.: $122-124{ }^{\circ} \mathrm{C}$; $\mathrm{R}_{f}=$ 0.34 in 40:60 ethyl acetate/hexane; FTIR (KBr): 3061, 2971, 2931, 2367, 1717, 1655, 1608, 1464, 1415, 1348, 1298, 1253, 1160, 1128, $1032 \mathrm{~cm}^{-1} ;{ }^{1} \mathrm{H}$ NMR $\left(400 \mathrm{MHz}, \mathrm{CDCl}_{3}\right): \delta 8.39(\mathrm{~d}, 1 \mathrm{H}, J=4.7 \mathrm{~Hz}), 7.47(\mathrm{t}$, $1 \mathrm{H}, J=7.8 \mathrm{~Hz}), 7.27(\mathrm{t}, 1 \mathrm{H}, J=7.3 \mathrm{~Hz}), 7.22(\mathrm{~d}, 1 \mathrm{H}$, $J=7.8 \mathrm{~Hz}), 7.16(\mathrm{~d}, 1 \mathrm{H}, J=8.0 \mathrm{~Hz}), 7.03-6.95(\mathrm{~m}$, $1 \mathrm{H}), 6.75$ (d, 1H, $J=7.4 \mathrm{~Hz}), 5.76(\mathrm{~s}, 1 \mathrm{H}), 4.29-4.09$ $(\mathrm{m}, 2 \mathrm{H}), 3.73(\mathrm{~s}, 2 \mathrm{H}), 3.19(\mathrm{~s}, 3 \mathrm{H}), 1.22(\mathrm{t}, 3 \mathrm{H}, J=7.0$ $\mathrm{Hz}) ;{ }^{13} \mathrm{C}\left\{{ }^{1} \mathrm{H}\right\}$ NMR $\left(100 \mathrm{MHz}, \mathrm{CDCl}_{3}\right): \delta$ 174.8, 170.0, 156.8, 149.3, 145.4, 136.2, 132.1, 128.6, 124.2, 122.1, 121.9, 120.1, 107.9, 62.1, 48.8, 35.0, 26.3, 14.1; HRMS: calcd. for $\mathrm{C}_{18} \mathrm{H}_{18} \mathrm{~N}_{2} \mathrm{O}_{3} \mathrm{~S}+\mathrm{Na}$ : 343.1111; found: 343.1123 .

3m: Red semi solid; yield: $73 \% ; \mathrm{R}_{f}=0.44$ in 30:70 ethyl acetate/hexane; FTIR (KBr): 3057, 2989, 2412, 2359, 2308, 1728, 1608, 1467, 1424, 1354, 1265, 1213, 1159, 1025, $967 \mathrm{~cm}^{-1}$; ${ }^{1} \mathrm{H}$ NMR (400 MHz, $\left.\mathrm{CDCl}_{3}\right): \delta 7.42-7.31(\mathrm{~m}, 2 \mathrm{H}), 7.30-7.19(\mathrm{~m}, 5 \mathrm{H}), 6.64$ $(\mathrm{d}, 1 \mathrm{H}, J=7.0 \mathrm{~Hz}), 4.84(\mathrm{~s}, 1 \mathrm{H}), 4.46$ and $4.41(\mathrm{AB} \mathrm{q}$, $2 \mathrm{H}, J=17.6 \mathrm{~Hz}), 4.26-4.09(\mathrm{~m}, 4 \mathrm{H}), 3.66$ and 3.39 $(\mathrm{ABq}, 2 \mathrm{H}, J=22.2 \mathrm{~Hz}), 1.27(\mathrm{t}, 3 \mathrm{H}, J=7.1 \mathrm{~Hz}), 1.19$ $(\mathrm{t}, 3 \mathrm{H}, J=7.1 \mathrm{~Hz}) ;{ }^{13} \mathrm{C}\left\{{ }^{1} \mathrm{H}\right\} \mathrm{NMR}\left(100 \mathrm{MHz}, \mathrm{CDCl}_{3}\right)$ : $\delta$ 174.5, 169.6, 167.6, 144.0, 133.4, 133.2, 132.4, $129.2,128.6,123.6,122.7,108.0,62.1,61.9,54.5$, 41.5, 34.5, 14.2, 14.1; HRMS: calcd. for $\mathrm{C}_{22} \mathrm{H}_{23} \mathrm{NO}_{5-}$ S+ H: 414.1370; found: 414.1379 .

3n: Red solid; yield: $82 \%$; M.p.: $112-114{ }^{\circ} \mathrm{C} ; \mathrm{R}_{f}=$ 0.48 in 30:70 ethyl acetate/hexane; FTIR (KBr): 3063 ,
2977, 2927, 2861, 2360, 1729, 1607, 1468, 1353, 1310, 1207, 1160, 1025, $969 \mathrm{~cm}^{-1}$; ${ }^{1} \mathrm{H}$ NMR (400 $\left.\mathrm{MHz}, \mathrm{CDCl}_{3}\right): \delta 7.37(\mathrm{~d}, 2 \mathrm{H}, J=6.8 \mathrm{~Hz}), 7.31-7.20$ $(\mathrm{m}, 4 \mathrm{H}), 6.67(\mathrm{~d}, 1 \mathrm{H}, J=8.4 \mathrm{~Hz}), 5.49(\mathrm{~s}, 1 \mathrm{H})$, $4.31-4.07(\mathrm{~m}, 2 \mathrm{H}), 3.77$ and $3.29(\mathrm{ABq}, 2 \mathrm{H}, J=22.3$ $\mathrm{Hz}), 3.17(\mathrm{~s}, 3 \mathrm{H}), 1.23(\mathrm{t}, 3 \mathrm{H}, J=7.1 \mathrm{~Hz}) ;{ }^{13} \mathrm{C}\left\{{ }^{1} \mathrm{H}\right\}$ NMR $\left(100 \mathrm{MHz}, \mathrm{CDCl}_{3}\right): \delta 174.4,168.8,144.4$, 134.0, 132.9, 131.3, 129.2, 129.1, 128.7, 127.7, 126.0, 108.7, 62.5, 52.9, 35.7, 26.4, 14.1; HRMS: calcd. for $\mathrm{C}_{19} \mathrm{H}_{18} \mathrm{ClNO}_{3} \mathrm{~S}+\mathrm{Na}$ : 398.0588; found: 398.0599 .

3o: Orange liquid; yield: $81 \% ; \mathrm{R}_{f}=0.50$ in $30: 70$ ethyl acetate/hexane; FTIR (KBr): 3063, 2974, 2929, $1721,1601,1465,1342,1291,1236,1151,1100$, 1026, $809 \mathrm{~cm}^{-1} ;{ }^{1} \mathrm{H}$ NMR (400 MHz, $\mathrm{CDCl}_{3}$ ): $\delta 7.46$ $(\mathrm{d}, 1 \mathrm{H}, J=8.5 \mathrm{~Hz}), 7.38(\mathrm{~d}, 2 \mathrm{H}, J=7.9 \mathrm{~Hz}), 7.30-7.20$ $(\mathrm{m}, 3 \mathrm{H}), 6.62(\mathrm{~d}, 1 \mathrm{H}, J=8.2 \mathrm{~Hz}), 5.54(\mathrm{~s}, 1 \mathrm{H})$, $4.27-4.12(\mathrm{~m}, 2 \mathrm{H}), 3.83$ and $3.36(\mathrm{ABq}, 2 \mathrm{H}, J=22.4$ $\mathrm{Hz}), 3.16(\mathrm{~s}, 3 \mathrm{H}), 1.22(\mathrm{t}, 3 \mathrm{H}, J=7.2 \mathrm{~Hz}) ;{ }^{13} \mathrm{C}\left\{{ }^{1} \mathrm{H}\right\}$ NMR $\left(100 \mathrm{MHz}, \mathrm{CDCl}_{3}\right): \delta 174.2,168.8,145.0$, 133.9, 133.2, 132.8, 132.4, 129.0, 128.6, 125.9, 117.6, 109.1, 62.4, 55.4, 35.7, 26.3, 14.1; HRMS: calcd. for $\mathrm{C}_{19} \mathrm{H}_{18} \mathrm{BrNO}_{3} \mathrm{~S}+\mathrm{Na}$ : 442.0083; found: 442.0085 .

3p: Red semi solid; yield: $74 \% ; \mathrm{R}_{f}=0.47$ in 30:70 ethyl acetate/hexane; FTIR (KBr): 3057, 2985, 2936, 2306, 1716, 1619, 1472, 1425, 1361, 1265, 1163, 740 $\mathrm{cm}^{-1} ;{ }^{1} \mathrm{H}$ NMR $\left(400 \mathrm{MHz}, \mathrm{CDCl}_{3}\right): \delta 7.35(\mathrm{~d}, 2 \mathrm{H}, J=$ $7.7 \mathrm{~Hz}), 7.28-7.22(\mathrm{~m}, 3 \mathrm{H}), 6.95(\mathrm{t}, 1 \mathrm{H}, J=9.4 \mathrm{~Hz})$, $6.66(\mathrm{dd}, 1 \mathrm{H}, J=3.8,8.5 \mathrm{~Hz}), 5.24(\mathrm{~s}, 1 \mathrm{H}), 4.26-4.14$ $(\mathrm{m}, 2 \mathrm{H}), 3.72$ and $3.31(\mathrm{ABq}, 2 \mathrm{H}, J=22.9 \mathrm{~Hz}), 3.17$ (s, 3H), $1.22(\mathrm{t}, 3 \mathrm{H}, J=7.1 \mathrm{~Hz}) ;{ }^{13} \mathrm{C}\left\{{ }^{1} \mathrm{H}\right\}$ NMR $(100$ $\left.\mathrm{MHz}, \mathrm{CDCl}_{3}\right): \delta 174.6,168.8,156.5(\mathrm{~d}, J=242.5 \mathrm{~Hz})$, $141.5,133.7,132.8,129.1,128.7,125.7$ (d, $J=3.4$ $\mathrm{Hz}), 121.7(\mathrm{~d}, J=16.6 \mathrm{~Hz}), 114.4(\mathrm{~d}, J=24.9 \mathrm{~Hz})$, $108.2(\mathrm{~d}, J=8.5 \mathrm{~Hz}), 62.5,49.0,35.6,26.4,14.1$; HRMS: calcd. for $\mathrm{C}_{19} \mathrm{H}_{18} \mathrm{FNO}_{3} \mathrm{~S}+\mathrm{Na}$ : 382.0884; found: 382.0893 .

3q: Orange solid; yield: $67 \%$; M.p.: $132-134{ }^{\circ} \mathrm{C} ; \mathrm{R}_{f}$ $=0.42$ in 40:60 ethyl acetate/hexane; FTIR (KBr): 3368, 3302, 2926, 2855, 2360, 1713, 1610, 1473, 1358, 1286, 1251, 1160, 1073, 1028, $747 \mathrm{~cm}^{-1} ;{ }^{1} \mathrm{H}$ NMR (400 MHz, $\left.\mathrm{CDCl}_{3}\right): \delta 8.17(\mathrm{~d}, 1 \mathrm{H}, J=8.3 \mathrm{~Hz})$; $7.39(\mathrm{~d}, 2 \mathrm{H}, J=7.6 \mathrm{~Hz}), 7.33-7.21(\mathrm{~m}, 3 \mathrm{H}), 6.82(\mathrm{~d}$, $1 \mathrm{H}, J=8.6 \mathrm{~Hz}), 5.26(\mathrm{~s}, 1 \mathrm{H}), 4.25(\mathrm{q}, 2 \mathrm{H}, J=7.0 \mathrm{~Hz})$, 3.58 and $2.97(\mathrm{ABq}, 2 \mathrm{H}, J=21.8 \mathrm{~Hz}), 3.23(\mathrm{~s}, 3 \mathrm{H})$, $1.26(\mathrm{t}, 3 \mathrm{H}, J=7.0 \mathrm{~Hz}) ;{ }^{13} \mathrm{C}\left\{{ }^{1} \mathrm{H}\right\} \mathrm{NMR}(100 \mathrm{MHz}$, $\left.\mathrm{CDCl}_{3}\right): \delta 174.0,168.3,149.7,142.3,134.1,133.8$, 130.8, 129.4, 129.1, 127.8, 125.9, 107.1, 62.7, 54.0, 35.0, 26.6, 14.1; HRMS: calcd. for $\mathrm{C}_{19} \mathrm{H}_{18} \mathrm{~N}_{2} \mathrm{O}_{5} \mathrm{~S}+$ Na: 409.0829; found: 409.0835 .

3r: Orange semi solid; yield: $70 \% ; \mathrm{R}_{f}=0.41$ in 40:60 ethyl acetate/hexane; FTIR (KBr): 3057, 2986, 2308, 1711, 1611, 1472, 1431, 1358, 1266, 1163, 1076, 1029, $897 \mathrm{~cm}^{-1} ;{ }^{1} \mathrm{H}$ NMR (400 MHz, $\left.\mathrm{CDCl}_{3}\right): \delta$ 
$7.34(\mathrm{~d}, 2 \mathrm{H}), 7.28-7.17(\mathrm{~m}, 3 \mathrm{H}), 6.75(\mathrm{~d}, 1 \mathrm{H}, J=8.4$ $\mathrm{Hz}), 6.66(\mathrm{~d}, 1 \mathrm{H}, J=8.4 \mathrm{~Hz}), 5.33(\mathrm{~s}, 1 \mathrm{H}), 4.18(\mathrm{q}, 2 \mathrm{H}$, $J=7.0 \mathrm{~Hz}), 3.70(\mathrm{~s}, 3 \mathrm{H}), 3.61$ and $3.12(\mathrm{ABq}, 2 \mathrm{H}, J=$ $22.0 \mathrm{~Hz}), 3.15(\mathrm{~s}, 3 \mathrm{H}), 1.21(\mathrm{t}, 3 \mathrm{H}, J=7.2 \mathrm{~Hz})$; ${ }^{13} \mathrm{C}\left\{{ }^{1} \mathrm{H}\right\}$ NMR $\left(100 \mathrm{MHz}, \mathrm{CDCl}_{3}\right): \delta 174.5,169.6$, 152.2, 138.1, 133.8, 133.7, 128.8, 128.3, 125.5, 123.3, 110.1, 107.7, 62.0, 56.4, 49.9, 35.4, 26.2, 14.2; HRMS: calcd. for $\mathrm{C}_{20} \mathrm{H}_{21} \mathrm{NO}_{4} \mathrm{~S}+\mathrm{H}$ : 372.1264; found: 372.1278 .

5a: Red semi solid; yield: $71 \% ; \mathrm{R}_{f}=0.51$ in $40: 60$ ethyl acetate/hexane; FTIR (Neat): 3426, 3064, 2984, 2252, 1709, 1608, 1470, 1349, 1301, 1251, 1184, 1131, 1027, 984, $912 \mathrm{~cm}^{-1}$; ${ }^{1} \mathrm{H}$ NMR (400 MHz, $\left.\mathrm{CDCl}_{3}\right): \delta 7.50-7.38(\mathrm{~m}, 2 \mathrm{H}), 7.31(\mathrm{t}, 1 \mathrm{H}, J=7.3 \mathrm{~Hz})$, $7.27(\mathrm{~m}, 4 \mathrm{H}), 6.76-6.66(\mathrm{~m}, 1 \mathrm{H}), 4.83(\mathrm{~s}, 1 \mathrm{H})$, 4.22-4.05 (m, 2H), 3.47 and $3.18(\mathrm{ABq}, 2 \mathrm{H}, J=22.1$ $\mathrm{Hz}), 3.18(\mathrm{~s}, 3 \mathrm{H}), 1.19(\mathrm{t}, 3 \mathrm{H}, J=7.1 \mathrm{~Hz}) ;{ }^{13} \mathrm{C}\left\{{ }^{1} \mathrm{H}\right\}$ NMR $\left(100 \mathrm{MHz}, \mathrm{CDCl}_{3}\right): \delta 174.5,170.3,145.2$, 136.0, 132.7, 129.1, 129.0, 128.4, 128.2, 123.5, 122.6, 107.6, 61.9, 45.9, 34.6, 26.4, 14.1; HRMS: calcd. for $\mathrm{C}_{19} \mathrm{H}_{19} \mathrm{NO}_{3} \mathrm{Se}+\mathrm{H}$ : 390.0603; found: 390.0608 .

5b: Red semi liquid; yield: $76 \% ; \mathrm{R}_{f}=0.55$ in 30:70 ethyl acetate/hexane; FTIR (Neat): 3431, 3061, 2935, 1718, 1618, 1469, 1228, 1125, 1025, 956, $811 \mathrm{~cm}^{-1}$; ${ }^{1} \mathrm{H}$ NMR (400 MHz, $\left.\mathrm{CDCl}_{3}\right): \delta 7.48-7.40(\mathrm{~m}, 2 \mathrm{H})$, $7.32(\mathrm{t}, 1 \mathrm{H}, J=7.4 \mathrm{~Hz}), 7.28-7.16(\mathrm{~m}, 5 \mathrm{H}), 6.71(\mathrm{~d}$, $1 \mathrm{H}, J=7.4 \mathrm{~Hz}), 4.85(\mathrm{~s}, 1 \mathrm{H}), 3.68(\mathrm{~s}, 3 \mathrm{H}), 3.47$ and $3.15(\mathrm{ABq}, 2 \mathrm{H}, J=22.2 \mathrm{~Hz}), 3.18(\mathrm{~s}, 3 \mathrm{H}) ;{ }^{13} \mathrm{C}\left\{{ }^{1} \mathrm{H}\right\}$ NMR $\left(100 \mathrm{MHz}, \mathrm{CDCl}_{3}\right): \delta 174.5,170.8,145.2$, 136.1, 132.7, 131.6, 129.2, 129.1, 128.5, 123.5, 122.7, 107.6, 52.9, 45.8, 34.6, 26.4; HRMS: calcd. for $\mathrm{C}_{18} \mathrm{H}_{16} \mathrm{NO}_{3} \mathrm{Se}+\mathrm{H}$ : 376.0446; found: 376.0448 .

5c: Red semi liquid; yield: $63 \% ; \mathrm{R}_{f}=0.40$ in $40: 60$ ethyl acetate/hexane; FTIR (Neat): 3433, 3057, 2978, 2932, 2333, 2091, 1730, 1606, 1466, 1355, 1207, 1025, 932, $871 \mathrm{~cm}^{-1} ;{ }^{1} \mathrm{H}$ NMR (400 MHz, $\left.\mathrm{CDCl}_{3}\right): \delta$ 7.49-7.39 (m, 2H), 7.36-7.29 (m, 1H), 7.28-7.18 (m, $4 \mathrm{H}), 6.60(\mathrm{~d}, 1 \mathrm{H}, J=7.3 \mathrm{~Hz}), 4.82(\mathrm{~s}, 1 \mathrm{H}), 4.43(\mathrm{~s}$, $2 \mathrm{H}), 4.22(\mathrm{q}, 2 \mathrm{H}, J=7.1 \mathrm{~Hz}), 4.18-4.08(\mathrm{~m}, 2 \mathrm{H}), 3.53$ and $3.19(\mathrm{ABq}, 2 \mathrm{H}, J=22.2 \mathrm{~Hz}), 1.27(\mathrm{t}, 3 \mathrm{H}, J=7.1$ $\mathrm{Hz}), 1.19(\mathrm{t}, 3 \mathrm{H}, J=7.0 \mathrm{~Hz}) ;{ }^{13} \mathrm{C}\left\{{ }^{1} \mathrm{H}\right\}$ NMR $(100$ $\left.\mathrm{MHz}, \mathrm{CDCl}_{3}\right): \delta 174.4,170.3,167.5,143.8,136.1$, 133.0, 131.5, 129.1 (8), 129.1 (1), 128.4, 123.2, 123.0, 107.6, 61.9, 61.8, 45.8, 41.4, 34.3, 14.2, 14.0; HRMS: calcd. for $\mathrm{C}_{22} \mathrm{H}_{24} \mathrm{NO}_{5} \mathrm{Se}+\mathrm{H}$ : 462.0814; found: 462.0814 .

5d: Red semi liquid; yield: $69 \% ; \mathrm{R}_{f}=0.51$ in 40:60 ethyl acetate/hexane; FTIR (Neat): 3419, 3055, 2980, 2930, 1955, 1717, 1605, 1462, 1340, 1229, 1162, 1026, $965 \mathrm{~cm}^{-1}$; ${ }^{1} \mathrm{H}$ NMR (400 $\left.\mathrm{MHz}, \mathrm{CDCl}_{3}\right): \delta$ 7.47-7.37 (m, 2H), 7.36-7.23 (m, 6H), 7.23-7.15 (m, $3 \mathrm{H}), 7.11(\mathrm{t}, 1 \mathrm{H}, J=7.9 \mathrm{~Hz}), 6.60(\mathrm{~d}, 1 \mathrm{H}, J=7.7 \mathrm{~Hz})$, 4.90 and $4.84(\mathrm{ABq}, 2 \mathrm{H}, J=15.6 \mathrm{~Hz}), 4.84(\mathrm{~s}, 1 \mathrm{H})$,
4.23-4.03 (m, 2H), 3.53 and $3.20(\mathrm{ABq}, 2 \mathrm{H}, J=22.2$ $\mathrm{Hz}), 1.19(\mathrm{t}, 3 \mathrm{H}, J=7.1 \mathrm{~Hz}) ;{ }^{13} \mathrm{C}\left\{{ }^{1} \mathrm{H}\right\}$ NMR $(100$ $\left.\mathrm{MHz}, \mathrm{CDCl}_{3}\right): \delta 174.5,170.3,144.1,136.2,135.8$, $132.8,129.1,128.8,128.3,128.1,127.7,127.4,123.5$, $122.6,108.5,61.9,45.7,43.8,34.5,14.1$; HRMS: calcd. for $\mathrm{C}_{25} \mathrm{H}_{23} \mathrm{NO}_{3} \mathrm{Se}+\mathrm{H}$ : 466.0916; found: 466.0918 .

5e: Red semi liquid; yield: $66 \% ; \mathrm{R}_{f}=0.51$ in $40: 60$ ethyl acetate/hexane; FTIR (Neat): 3407, 3059, 2926, $2858,1720,1602,1465,1345,1302,1224,1134$, 1027, $806 \mathrm{~cm}^{-1}$; ${ }^{1} \mathrm{H}$ NMR (400 MHz, $\left.\mathrm{CDCl}_{3}\right): \delta$ 7.48-7.38 (m, 3H), 7.34-7.27 (m, 1H), 7.24-7.15 (m, $2 \mathrm{H}), 6.57(\mathrm{~d}, 1 \mathrm{H}, J=8.3 \mathrm{~Hz}), 5.58(\mathrm{~s}, 1 \mathrm{H}), 4.28-4.09$ $(\mathrm{m}, 2 \mathrm{H}), 3.69$ and $3.17(\mathrm{ABq}, 2 \mathrm{H}, J=21.9 \mathrm{~Hz}), 3.19$ $(\mathrm{s}, 2 \mathrm{H}), 1.24$ (s, 3H, $J=7.1 \mathrm{~Hz}) ;{ }^{13} \mathrm{C}\left\{{ }^{1} \mathrm{H}\right\}$ NMR $(100$ $\left.\mathrm{MHz}, \mathrm{CDCl}_{3}\right): \delta 174.2,169.3,144.8,136.5,133.1$, 132.4, 129.1, 129.0, 127.8, 126.1, 117.7, 108.8, 62.5, 47.0, 35.8, 26.3, 14.1; HRMS: calcd. for $\mathrm{C}_{19} \mathrm{H}_{18-}$ $\mathrm{BrNO}_{3} \mathrm{Se}+\mathrm{H}: 467.9708$; found: 467.9704 .

5f: Red semi liquid; yield: $71 \% ; \mathrm{R}_{f}=0.51$ in 40:60 ethyl acetate/hexane; FTIR (Neat): 3454, 3061, 2934, 1718, 1618, 1472, 1347, 1289, 1236, 1125, 1026, 957 $\mathrm{cm}^{-1} ;{ }^{1} \mathrm{H}$ NMR (400 MHz, $\left.\mathrm{CDCl}_{3}\right): \delta 7.41(\mathrm{~d}, 2 \mathrm{H}, J=$ $7.6 \mathrm{~Hz}), 7.33-7.24(\mathrm{~m}, 1 \mathrm{H}), 7.18(\mathrm{t}, 2 \mathrm{H}, J=7.3 \mathrm{~Hz})$, $6.90(\mathrm{t}, 1 \mathrm{H}, J=9.6 \mathrm{~Hz}), 6.62(\mathrm{dd}, 1 \mathrm{H}, J=8.4,3.7 \mathrm{~Hz})$, $5.27(\mathrm{~s}, 1 \mathrm{H}), 4.20(\mathrm{q}, 2 \mathrm{H}, J=6.9 \mathrm{~Hz}), 3.58$ and 3.13 $(\mathrm{ABq}, 2 \mathrm{H}, J=22.2 \mathrm{~Hz}), 3.16(\mathrm{~s}, 3 \mathrm{H}), 1.23(\mathrm{t}, 3 \mathrm{H}, J=$ $7.0 \mathrm{~Hz}) ;{ }^{13} \mathrm{C}\left\{{ }^{1} \mathrm{H}\right\}$ NMR $\left(100 \mathrm{MHz}, \mathrm{CDCl}_{3}\right): \delta 174.5$, $169.3,156.7(\mathrm{~d}, J=242.7 \mathrm{~Hz}) 141.3,136.3,129.1$, $127.9,125.7,122.3,114.3(\mathrm{~d}, J=25.2 \mathrm{~Hz}), 107.8(\mathrm{~d}$, $J=8.7 \mathrm{~Hz}), 62.4,40.5,35.5,26.4$, 14.1; HRMS: calcd. for $\mathrm{C}_{19} \mathrm{H}_{18} \mathrm{FNO}_{3} \mathrm{Se}+\mathrm{H}$ : 407.0436; found: 407.0439.

Synthesis of sulfone 6: $m$-CPBA $(121 \mathrm{mg}, 0.7036$ $\mathrm{mmol}$ ) was added to a solution of oxindole $\mathbf{3}$ (80 g, $0.2345 \mathrm{mmol})$ in $\mathrm{CH}_{2} \mathrm{Cl}_{2}(10 \mathrm{~mL})$ in $25 \mathrm{~mL}$ round bottom flask. The reaction mixture was stirred at room temperature for $2 \mathrm{~h}$ and analyzed by TLC. After completion, the reaction mixture was diluted with $\mathrm{CH}_{2} \mathrm{Cl}_{2}$ $(10 \mathrm{~mL})$ and neutralized by aq. $\mathrm{NaHCO}_{3}$. The organic layer was separated and evaporated under the reduced pressure. The resultant residue was purified by column chromatography using EtOAc/hexane as eluent to give sulfone $\mathbf{6}$ as green semi-liquid in $78 \%$ yield. $\mathrm{R}_{f}=0.38$ in 40:70 ethyl acetate/hexane; FTIR (Neat): 3426, 3064, 2984, 2252, 1709, 1608, 1470, 1349, 1301, 1251, 1184, 1131, 1027, 984, $912 \mathrm{~cm}^{-1}$; ${ }^{1} \mathrm{H}$ NMR $(400 \mathrm{MHz}$, $\left.\mathrm{CDCl}_{3}\right): \delta 7.73-7.59(\mathrm{~m}, 3 \mathrm{H}), 7.47(\mathrm{~d}, 2 \mathrm{H}, J=7.6 \mathrm{~Hz})$, $7.22(\mathrm{~m}, 1 \mathrm{H}, J=7.8 \mathrm{~Hz}), 7.15(\mathrm{~d}, 1 \mathrm{H}, J=8.0 \mathrm{~Hz}), 6.83(\mathrm{~d}$, $1 \mathrm{H}, J=7.6 \mathrm{~Hz}), 5.09$ (s, 1H), 4.32-4.06 (m, 2H), 3.46 and $3.37(\mathrm{ABq}, 2 \mathrm{H}, J=22.0 \mathrm{~Hz}), 3.19(\mathrm{~s}, 3 \mathrm{H}), 1.21(\mathrm{t}$, $3 \mathrm{H}, J=7.1 \mathrm{~Hz}) ;{ }^{13} \mathrm{C}\left\{{ }^{1} \mathrm{H}\right\}$ NMR $\left(100 \mathrm{MHz}, \mathrm{CDCl}_{3}\right): \delta$ $174.0,164.3,145.5,136.6,134.5,130.0,128.8,128.4$, $126.3,124.3,123.2,109.2,72.3,62.8,34.8,26.4,13.9$; 
HRMS: calcd. for $\mathrm{C}_{18} \mathrm{H}_{18} \mathrm{NO}_{5} \mathrm{~S}+\mathrm{H}$ : 360.0900 ; found: 360.0890 .

\section{Results and Discussion}

Initially, $\alpha$-thioester 1a derived from thiophenol and 3-diazoindolin-2-one $\mathbf{2 a}$ derived from isatins, were chosen as model substrates to study the rhodium-catalyzed Sommelet-Hauser type rearrangement (Table 1). Thus, one equivalent of $\alpha$-thioester 1a was treated with one equivalent of 3-diazoindolin-2-one $\mathbf{2 a}$ in the presence of $2 \mathrm{~mol} \%$ of rhodium acetate in toluene at $120{ }^{\circ} \mathrm{C}$. To our delight, the formation of expected C4-thioalkylated oxindole product 3a was observed in $76 \%$ yield (Table 1, entry 1). The formation and structure of oxindole $\mathbf{3 a}$ was unambiguously confirmed by X-ray analysis (Figure 2). ${ }^{22}$ After the successful confirmation of the product, attempts were devoted to optimize the reaction. The use of DCM as a solvent at $40{ }^{\circ} \mathrm{C}$ with equimolar amount of 1a and 2a also afforded the expected product $\mathbf{3 a}$ in comparable yield within $4 \mathrm{~h}$ (Table 1 , entry 2 ). Next, the reaction temperature was decreased from $40{ }^{\circ} \mathrm{C}$ to room temperature in $\mathrm{DCM}$, which gave the product $\mathbf{3 a}$ in decreased yield (Table 1, entry 3). Interestingly, increasing the amount of $\mathbf{2 a}$ in DCM at room temperature showed significant improvement in the reaction (Table 1, entry 4 and 5). The best yield of $81 \%$ of 3a was observed with two equivalents of $\mathbf{2 a}$.

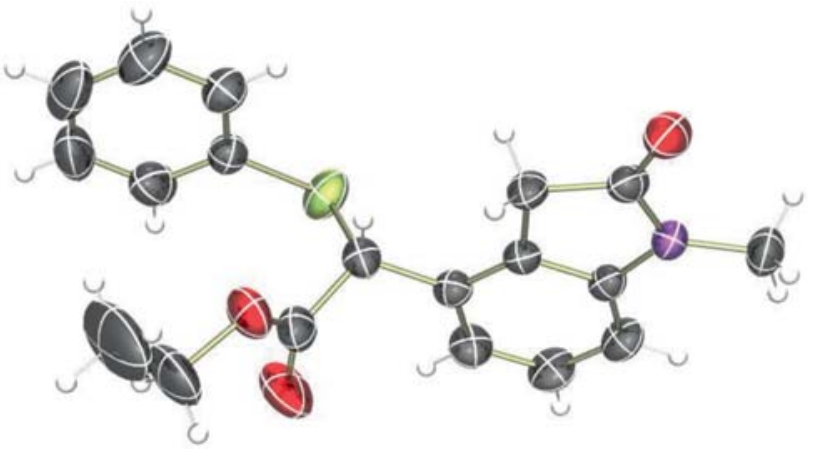

Figure 2. ORTEP diagram of oxindole $3 \mathrm{a}$.

On the other hand, the reaction of two equivalents of $\mathbf{1 a}$ and one equivalent of $\mathbf{2 a}$ gave only $59 \%$ of $3 \mathrm{a}$ (Table 1, entry 6). Keeping the equivalents of reagents, DCM and room temperature as a constant, decrease the catalyst loading from $2 \mathrm{~mol} \%$ to $1 \mathrm{~mol} \%$ gave the product 3a in diminished yield (Table 1, entry 7). Furthermore, replacing the catalyst $\mathrm{Rh}_{2}(\mathrm{OAc})_{4}$ with $\mathrm{Rh}_{2}(\mathrm{Oct})_{4}$ also led to the formation of product $\mathbf{3 a}$ in comparable yield (Table 1, entry 8). Similarly, changing the solvent from DCM to chloroform also gave the product in $78 \%$ yield (Table 1, entry 9). From all the above optimization studies, following conditions were chosen for studying the scope and generality of the present Sommelet-Hauser type rearrangement: $\alpha$-thioester 1a (1 equiv), 3-diazoindolin-2-one 2a (2 equiv), $\mathrm{Rh}_{2}(\mathrm{OAc})_{4}(2 \mathrm{~mol} \%)$, dichloromethane, rt, $2 \mathrm{~h}$.

Table 1. Rhodium-catalyzed Sommelet-Hauser type rearrangemen: optimization studies ${ }^{\mathrm{a}}$

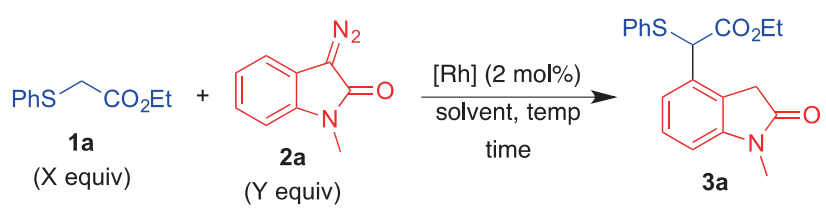

\begin{tabular}{lcclccc}
\hline Entry & 1a (X equiv) & 2a (Y equiv) & Solvent & Temp $\left({ }^{\circ} \mathrm{C}\right)$ & Time $(\mathrm{h})$ & Yield $(\%)^{\mathrm{b}}$ \\
\hline 1 & 1 & 2 & Toluene & 120 & 6 & 76 \\
2 & 1 & 1 & $\mathrm{CH}_{2} \mathrm{Cl}_{2}$ & 40 & 4 & 72 \\
3 & 1 & 1 & $\mathrm{CH}_{2} \mathrm{Cl}_{2}$ & $\mathrm{rt}$ & 4 & 61 \\
4 & 1 & 1.5 & $\mathrm{CH}_{2} \mathrm{Cl}_{2}$ & $\mathrm{rt}$ & 2 & 70 \\
5 & 1 & 2 & $\mathrm{CH}_{2} \mathrm{Cl}_{2}$ & $\mathrm{rt}$ & 2 & 81 \\
6 & 2 & 1 & $\mathrm{CH}_{2} \mathrm{Cl}_{2}$ & $\mathrm{rt}$ & 2 & 59 \\
$7^{\text {c }}$ & 1 & 2 & $\mathrm{CH}_{2} \mathrm{Cl}_{2}$ & $\mathrm{rt}$ & 2 & 68 \\
$8^{\text {d }}$ & 1 & 2 & $\mathrm{CH}_{2} \mathrm{Cl}_{2}$ & $\mathrm{rt}$ & 2 & 80 \\
9 & 1 & 2 & $\mathrm{CHCl}_{3}$ & $\mathrm{rt}$ & 2 & 78 \\
\hline
\end{tabular}

${ }^{a}$ Reaction conditions: $\alpha$-thioester $1 \mathbf{a}$ (X equiv), 3-diazoindolin-2-one $\mathbf{2 a}$ (Y equiv), $\mathrm{Rh}_{2}(\mathrm{OAc})_{4}$ (2 mol\%), solvent, temp, time

b Isolated yields

c $\mathrm{Rh}_{2}(\mathrm{OAc})_{4}(1 \mathrm{~mol} \%)$ was used

d $\mathrm{Rh}_{2}(\mathrm{Oct})_{4}(2 \mathrm{~mol} \%)$ was used. 
Having found the best-optimized conditions, next, the generality of the developed transformation was examined with respect to various $\alpha$-thioester 1 . At first, scope of various arene substitutions in $\alpha$-thioester was investigated. As can be seen in Scheme 2, all the substituted $\alpha$-thioester $\mathbf{1}$ afforded the corresponding oxindoles $\mathbf{3}$ in good yield. For instance, replacing of phenyl with electron-rich arene moieties such as 4-methoxy and 2,3-dimethoxyphenyl substituted $\alpha$-thioesters afforded corresponding C4-thioalkylated oxindoles $\mathbf{3 b}$ and $\mathbf{3 d}$ in $\sim 70 \%$ yields under the optimized conditions. Similarly, the present method was successfully applied to the haloarene-substituted $\alpha$-thioesters for the synthesis of synthetically useful halo substituted oxindoles $\mathbf{3 e - 3} \mathbf{3}$ in excellent yields.

Importantly, sterically hindered ortho-substituted 2,4-dimethylphenyl substituted $\alpha$-thioesters underwent smooth reaction with $\mathbf{2 a}$ to provide the corresponding oxindole $3 \mathbf{c}$ in $64 \%$ yield. Interestingly, acid-sensitive, silyloxyarene substituted $\alpha$-thioester was also welltolerated under the optimized conditions to give oxindole $\mathbf{3 h}$ in $81 \%$ yield. Furthermore, $\alpha$-thioester derived from pyridine-2-thiol also worked well under the optimized conditions to furnish oxindole $\mathbf{3 i}$ in $88 \%$ yield. On the other hand, the replacement of ester moiety with other electron-withdrawing groups, such as methyl ketone, carboxamide and nitro group, failed to afford the expected $\mathrm{C} 4$-substituted product $\mathbf{3 j} \mathbf{j} \mathbf{3}$. This is possibly due to the less acidity of $\mathrm{C}-\mathrm{H}$ bond in carboxamide and high stability of the anion generated from nitro derivatives.

Successively, the scope of 3-diazoindolin-2-one 2 was also examined under the present optimized conditions (Scheme 3). $N$-substituted diazo derivatives such as methyl and functionalized alkyl group were subjected under rhodium-catalyzed reaction with 1a, which led to the formation of oxindole products $\mathbf{3 a}$ and $\mathbf{3 m}$ in $81 \%$ and $73 \%$ yields, respectively. C4-Functionalized chloro, bromo and fluoro substituted oxindole $\mathbf{3 n}-\mathbf{3 p}$ were achieved in good yield from corresponding substituted diazo compounds. It is important to note that both electron-withdrawing, 5-nitro-substituted and electron-donating, 5-methoxy-substituted diazo derivatives underwent smooth reaction to afford the oxindole products $\mathbf{3 q}$ and $\mathbf{3 r}$ in good yields. Unfortunately, the replacement of 3-diazoindolin-2-one 2 with cyclic diazoketone $\mathbf{2}$ s under the optimized conditions failed to afford the corresponding product $\mathbf{3 s}$.

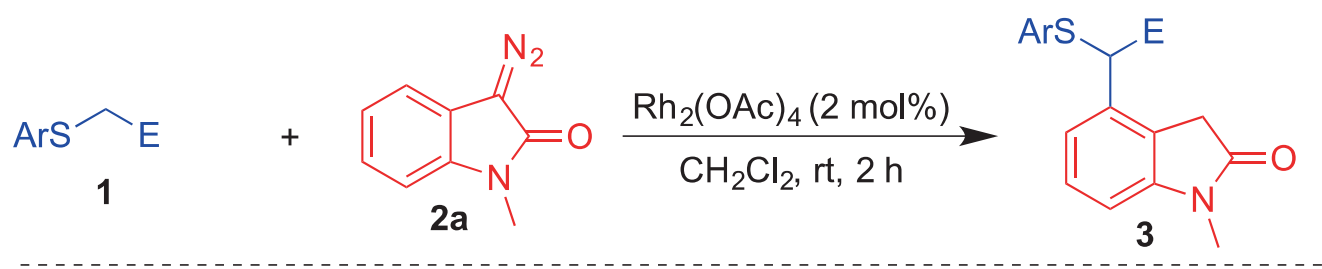<smiles>CCOC(=O)C(Sc1ccc(OC)cc1)c1cccc2c1CC(=O)N2C</smiles><smiles>CCOC(=O)C(Sc1ccc(C)cc1C)c1cccc2c1CC(=O)N2C</smiles><smiles>CCOC(=O)Cc1cccc2c1CC(=O)N2C</smiles><smiles>CCOC(=O)C(Sc1ccccc1Cl)c1cccc2c1CC(=O)N2C</smiles><smiles>CCOC(=O)C(Sc1ccccc1F)c1cccc2c1CC(=O)N2C</smiles><smiles>CCOC(=O)C(Sc1ccc(Cl)cc1)c1cccc2c1CC(=O)N2C</smiles><smiles>CCOC(=O)C(Sc1ccc(O[Si](C)(C)C(C)(C)C)cc1)c1cccc2c1CC(=O)N2C</smiles><smiles>CCOC(=O)C(Sc1ccccn1)c1cccc2c1CC(=O)N2C</smiles>

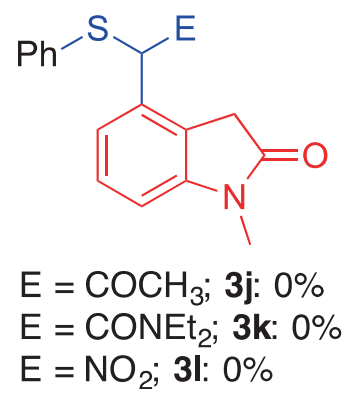

Scheme 2. Synthesis of C4-thioalkylated oxindoles 3: Scope and limitation of $\alpha$-thioester 1 . 
<smiles>CCOC(=O)CSc1ccccc1</smiles>

$3 a: 81 \%$<smiles>CCOC(=O)C(Sc1ccccc1)c1c(F)ccc2c1CC(=O)N2C</smiles>

3p: $74 \%$<smiles></smiles>

2

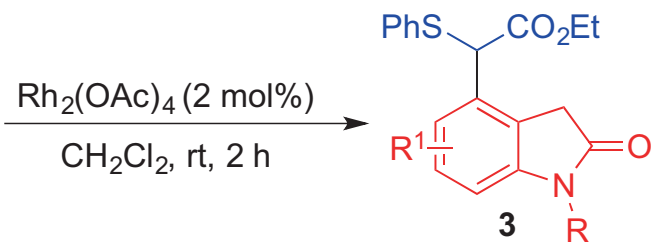<smiles>CCOC(=O)C(Sc1ccccc1)c1c(Cl)ccc2c1CC(=O)N2C</smiles>

$3 n: 82 \%$<smiles>CCOC(=O)C(c1ccccc1)c1c(OC)ccc2c1CC(=O)N2C</smiles>

3r: $70 \%$<smiles>CCOC(=O)C(c1ccccc1)c1c(Br)ccc2c1CC(=O)N2C</smiles>

3o: $81 \%$<smiles>CCOC(=O)C(c1ccccc1)c1cccc2c1CC(=O)c1ccccc1-2</smiles>

Scheme 3. Synthesis of C4-thioalkylated oxindoles 3: Scope and limitation of diazo compound 2.

After the successful development of SommeletHauser type rearrangement of 3-diazoindolin-2-one with $\alpha$-thioester, next, the extension of the present reaction to $\alpha$-selenoesters was envisaged for the synthesis of C4-selenoalkylated oxindole derivatives. Thus, one equivalent of $\alpha$-selenoester $\mathbf{4 a}$ was subjected to two equivalents of diazo compound 2a in the presence of $2 \mathrm{~mol} \%$ of $\mathrm{Rh}_{2}(\mathrm{OAc})_{4}$ in dichloromethane at room temperature (Scheme 4). As expected, C4selenoalkylated oxindole 5a was observed in $71 \%$ yield. This implies that the developed reaction works equally well for selenium-ylide derived the $\alpha$-selenoesters. Further, the scope of $\alpha$-selenoesters with various $N$-substituted diazo derivatives as examined. Replacement of ethyl ester with methyl ester in $\alpha$ selenoesters gave the oxindole $\mathbf{5} \mathbf{b}$ in comparable yield. The reaction of functionalized alkyl and benzyl group containing diazo compound under standard conditions led to the formation of oxindole products $\mathbf{5 c}$ and $\mathbf{5 d}$ in $63 \%$ and $69 \%$ yields, respectively. Similarly, halo substituted oxindoles 5e-5f were also synthesized in good yield from corresponding diazo compounds. Unfortunately, $\alpha$-selenoesters derived from methyl 2-bromopropionate did not afford the expected oxindole $\mathbf{5 g}$, possibly due to the steric hindrance.

After the successful demonstration of generality of the developed method, chemoselectivity of SommeletHauser type rearrangement was examined with $\alpha$-thioesters and $\alpha$-selenoesters (Scheme 5). The

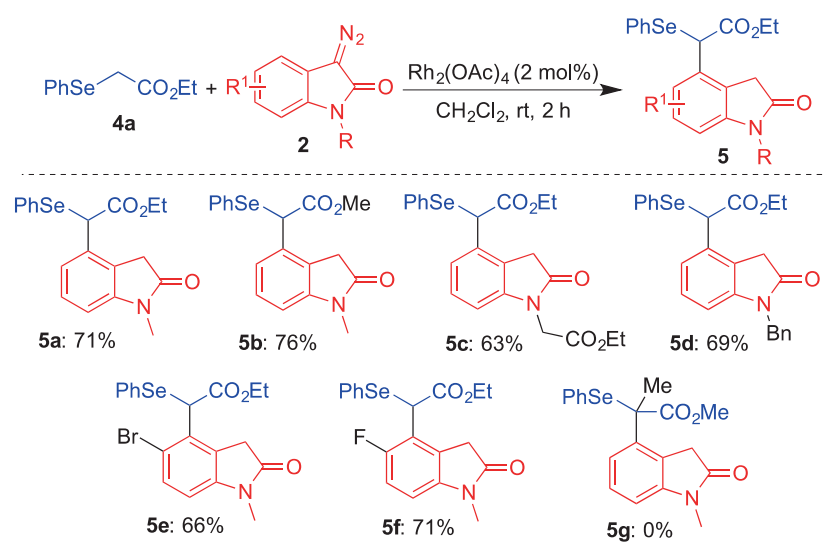

Scheme 4. Synthesis of C4-selenoalkylated oxindoles 5.

reaction of equimolar mixture of $\mathbf{1 a}$ and $\mathbf{4 b}$ with diazo compound 2a in the presence of $2 \mathrm{~mol} \%$ of rhodium acetate in DCM afforded the mixture of oxindoles $\mathbf{3 a}$ and $5 \mathrm{e}$ in $59 \%$ overall yield with 1:1.2 ratio (Scheme 5a). This observation suggests that reactivity of $\alpha$-selenoester towards Sommelet-Hauser type rearrangement under the developed conditions is significantly higher compared to $\alpha$-thioesters. Next, the possible synthetic conversion of oxindole $\mathbf{3 a}$ was also investigated (Scheme 5b). The sulfur moiety in the synthesized oxindole $\mathbf{3 a}$ was readily oxidized in the presence of $m$-CPBA at room temperature in $\mathrm{CH}_{2} \mathrm{Cl}_{2}$ to corresponding sulfone $\mathbf{6}$ in $78 \%$ yield, which could be readily applied for subsequent synthetic manipulation. 


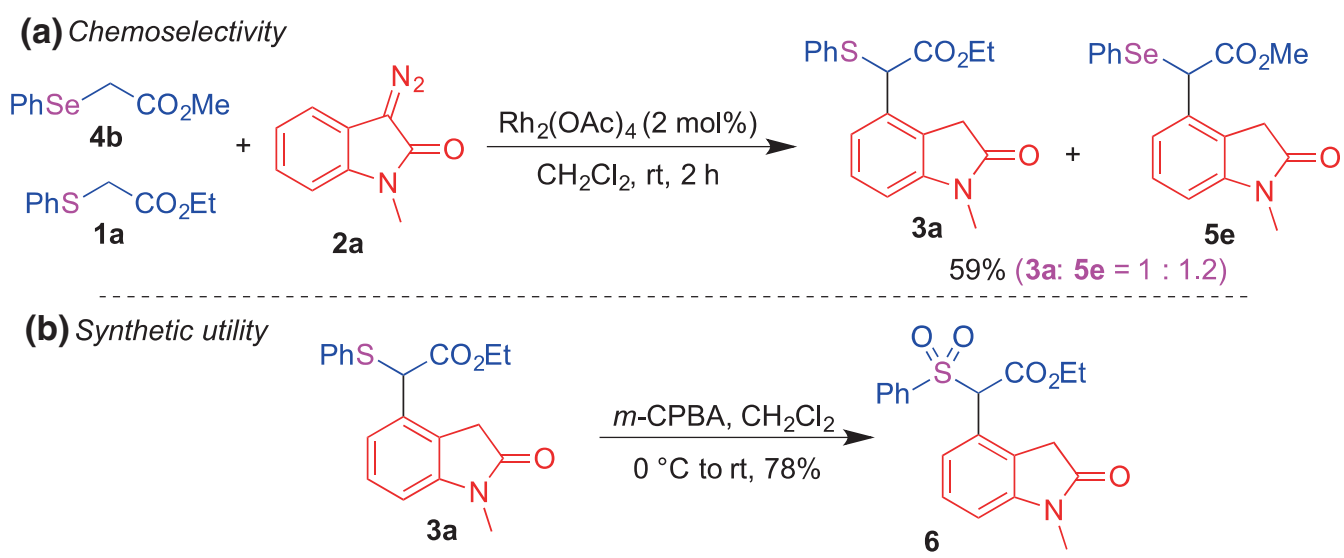

Scheme 5. Chemoselectivity and synthetic utility.

Having demonstrated the efficient synthesis of C4chalcogenoalkylated oxindoles, based on the literature precedence, the plausible mechanism for the rhodiumcatalyzed transformation was postulated (Scheme 6). The reaction starts with the generation of reactive rhodium carbenoid $\mathbf{A}$ from diazo compound $\mathbf{2}$ and $\mathrm{Rh}_{2}(\mathrm{OAc})_{4}$. Subsequent trapping of formed rhodium carbenoid A with $\alpha$-thioester $/ \alpha$-selenoester $\mathbf{1} / \mathbf{4}$ via nucleophilic attached would generate the metal-bound S/Se-ylide B. Regeneration of rhodium catalyst from metal-bound ylide $\mathbf{B}$ would form the $\mathrm{S} / \mathrm{Se}$-ylide $\mathbf{B}^{\prime}$. Intramolecular proton transfer in $\mathbf{B}^{\prime}$ would provide rearranged ylide $\mathbf{C}$. Formation of enone intermediate D could be readily realized through the [2, 3]-sigmatropic rearrangement of resultant ylide C. Finally, the expected oxindole product $\mathbf{3 / 5}$ could be achieved via the aromatization of intermediate $\mathbf{D}$.

In conclusion, a general and efficient synthesis of C4-thioalkylated oxindoles has been accomplished through rhodium-catalyzed Sommelet-Hauser type rearrangement of $\alpha$-thioester with 3-diazoindolin-2one. The developed reaction tolerates various reactive functional groups and allowed the selective synthesis of various C4-thioalkylated oxindoles in good yield to excellent yield. Furthermore, the developed reaction was successfully extended to $\alpha$-selenoester for the synthesis of C4-selenoalkylated oxindoles. Importantly, the chemoselective experiment suggested the significant higher reactivity of $\alpha$-selenoesters towards metal carbenoids compared to $\alpha$-thioesters.

\section{Supplementary Information (SI)}

${ }^{1} \mathrm{H}$ and ${ }^{13} \mathrm{C}$ NMR spectra of isolated compounds and CIF file containing crystallographic information of compound $3 \mathrm{a}$ are provided in the supporting information. Supplementary information is available at www.ias.ac.in/chemsci.

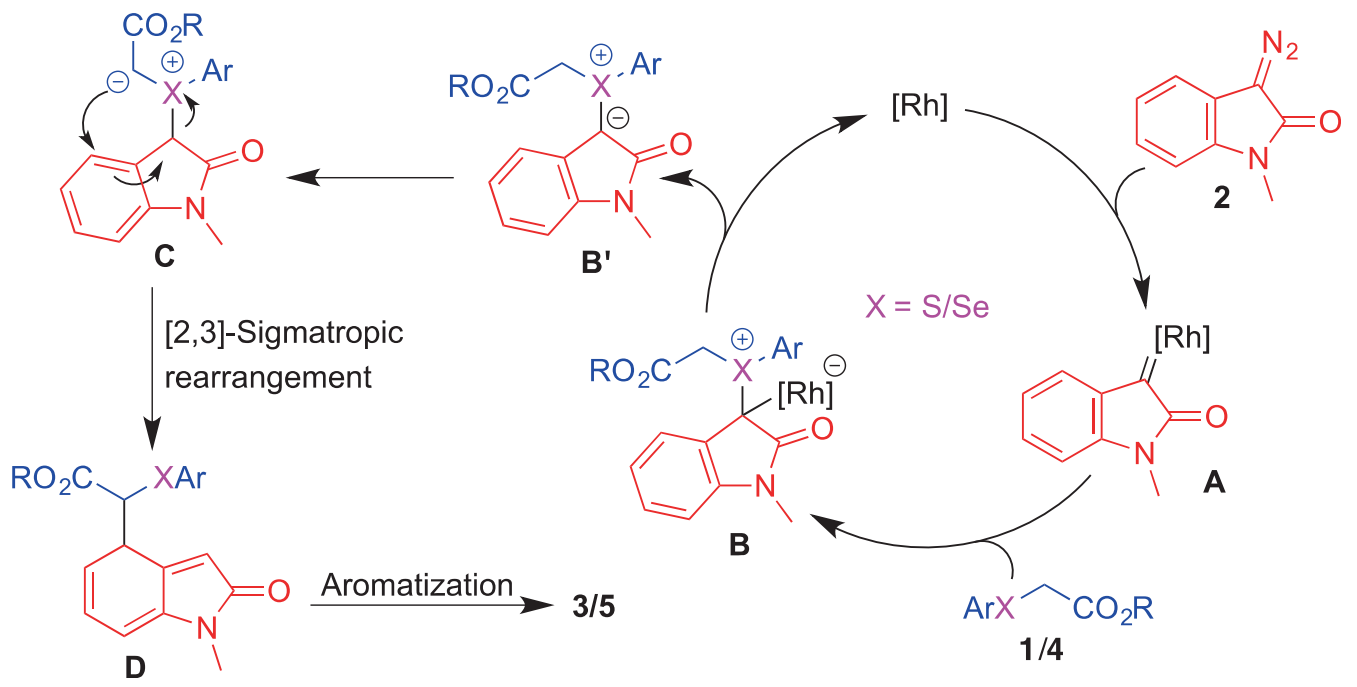

Scheme 6. A plausible mechanism. 


\section{Acknowledgements}

We thank DST-SERB (EMR/2016/003677) for the financial support and DST-FIST for ESI-MS facility. ACSR thanks the Council of Scientific \& Industrial Research (CSIR) for fellowship.

\section{References}

1. (a) Silva B V 2013 Isatin, a versatile molecule: studies in Brazil J. Braz. Chem. Soc. 24 707; (b) Manzoli L, Flacco M E, Boccia S, D'Andrea E, Panic N, Marzuillo C, Siliquini R, Ricciardi W, Villari P and Ioannidis J P A 2016 Generic versus brand-name drugs used in cardiovascular diseases Eur. J. Epidemiol. 31351

2. Bazoui H, Zahouily $M$, Boulajaaj S, Sebti $S$ and Zakarya D 2002 QSAR for anti-HIV activity of HEPT derivatives SAR QSAR Environ. Res. 13567

3. (a) Tanaka S, Amling M, Neff L, Peyman A, Uhlmann E, Levy J B and Baron R 1996 c-Cbl is downstream of $\mathrm{c}-\mathrm{Src}$ in a signalling pathway necessary for bone resorption Nature 383 528; (b) Hoffman R, Dennis I F and Donaldson J 1995 Protein binding modulates inhibition of the epidermal growth factor receptor kinase and DNA synthesis by tyrphostins Cancer Chemother. Pharmacol. 36316

4. Renslo A R, Jaishankar P, Venkatachalam R, Hackbarth C, Lopez S, Patel D V and Gordeev M F 2005 Conformational Constraint in Oxazolidinone Antibacterials. Synthesis and Structure-Activity Studies of (Azabicyclo[3.1.0] hexylphenyl)oxazolidinones $\mathrm{J}$. Med. Chem. 485009

5. Natarajan A, Guo Y, Harbinski F, Fan Y-H, Chen H, Luus L, Diercks J, Aktas H, Chorev M and Halperin J A 2004 Novel Arylsulfoanilide-Oxindole Hybrid as an Anticancer Agent That Inhibits Translation Initiation $J$. Med. Chem. 474979

6. (a) Gallagher G, Lavanchy P G, Wilson J W, Hieble J P and DeMarinis R M 1985 4-[2-(Di-n-propylamino)ethyl]-2(3H)-indolone: a prejunctional dopamine receptor agonist J. Med. Chem. 28 1533; (b) Kikuchi C, Hiranuma T and Koyama M 2002 Tetrahydrothienopyridylbutyl-tetrahydrobenzindoles: New Selective Ligands of the 5-HT7 Receptor Bioorg. Med. Chem. Lett. 12 2549; (c) Tokunaga T, Hume W E, Umezome T, Okazaki K, Ueki Y, Kumagai K, Hourai S, Nagamine J, Seki H, Taiji M, Noguchi H and Nagata R 2001 Oxindole Derivatives as Orally Active Potent Growth Hormone Secretagogues J. Med. Chem. 44 4641

7. (a) Dalpozzo R 2017 Catalytic asymmetric synthesis of hetero-substituted oxindoles Org. Chem. Front. 4 2063; (b) Dalpozzo R, Bartoli G and Bencivenni G 2012 Recent advances in organocatalytic methods for the synthesis of disubstituted 2- and 3-indolinones Chem. Soc. Rev. 417247

8. (a) Yang B H and Buchwald S L 1999 The Development of Efficient Protocols for the Palladium-Catalyzed Cyclization Reactions of Secondary Amides and Carbamates Org. Lett. 1 35; (b) van den Hoogenband A,
Lange J H M, Iwema-Bakker W I, den Hartog J A J, van Schaik J, Feenstra R W and Terpstra J W 2006 An efficient one-pot synthesis of novel 4-aryl-1-methyloxindoles Tetrahedron Lett. 47 4361; (c) Poondra R R and Turner N J 2005 Microwave-Assisted Sequential Amide Bond Formation and Intramolecular Amidation: A Rapid Entry to Functionalized Oxindoles $\mathrm{Org}$. Lett. 7 863; (d) Dounay A B, Hatanaka K, Kodanko J J, Oestreich M, Overman L E, Pfeifer L A and Weiss M M 2003 Catalytic Asymmetric Synthesis of Quaternary Carbons Bearing Two Aryl Substituents. Enantioselective Synthesis of 3-Alkyl-3-Aryl Oxindoles by Catalytic Asymmetric Intramolecular Heck Reactions $J$. Am. Chem. Soc. 125 6261; (e) Correia V G, Abreu J C, Barata C A E and Andrade L H 2017 Iron-Catalyzed Synthesis of Oxindoles: Application to the Preparation of Pyrroloindolines Org. Lett. 19 1060; (f) Pang Y, Guan M, Zeng R and Zhao Y 2017 A practical approach for the synthesis of oxindole and isatin derivatives by Pd-catalyzed intramolecular amination Org. Chem. Front. 42408

9. Abe T, Kosaka Y, Asano M, Harasawa N, Mishina A, Nagasue M, Sugimoto Y, Katakawa K, Sueki S, Anada M and Yamada K 2019 Direct C4-Benzylation of Indoles via Tandem Benzyl Claisen/Cope Rearrangements Org. Lett. 21826

10. Ye T and McKervey M A 1994 Organic Synthesis with a-Diazo Carbonyl Compounds Chem. Rev. 941091

11. Davies H M L and Beckwith R E J 2003 Catalytic Enantioselective $\mathrm{C}-\mathrm{H}$ Activation by Means of Metal-Carbenoid-Induced C-H Insertion Chem. Rev. 1032861

12. (a) Gillingham D and Fei N 2013 Catalytic X-H insertion reactions based on carbenoids Chem. Soc. Rev. 42 4918; (b) Guttenberger N and Breinbauer R $2017 \mathrm{C}-\mathrm{H}$ and $\mathrm{C}-\mathrm{C}$ bond insertion reactions of diazo compounds into aldehydes Tetrahedron $\mathbf{7 3} 6815$

13. Ford A, Miel H, Ring A, Slattery C N, Maguire A R and McKervey M A 2015 Modern Organic Synthesis with $\alpha$ Diazocarbonyl Compounds Chem. Rev. 1159981

14. (a) Aggarwal V K and Winn C L 2004 Catalytic, Asymmetric Sulfur Ylide-Mediated Epoxidation of Carbonyl Compounds: Scope, Selectivity, and Applications in Synthesis Acc. Chem. Res. 37 611; (b) Neuhaus J D, Oost R, Merad J and Maulide N 2018 Sulfur-Based Ylides in Transition-Metal-Catalysed Processes Top. Curr. Chem. 37615

15. (a) Tayama E 2016 Ring-Substitution, Enlargement, and Contraction by Base-Induced Rearrangements of N-Heterocyclic Ammonium Salts Heterocycles 92 793; (b) Tayama E 2015 Recent Advances in the Base-Induced Sommelet-Hauser Rearrangement of Amino Acid Derived Ammonium Ylides Chem. Rec. 15 789; (c) Roy T, Gaykar R N, Bhattacharjee S and Biju A T 2019 The aryne Sommelet-Hauser rearrangement Chem. Commun. 553004

16. (a) Vedejs E and West F G 1986 Ylides by the desilylation of $\alpha$-silyl onium salts Chem. Rev. 86 941; (b) Dormann K L and Brückner R 2007 Variable Synthesis of the Optically Active Thiotetronic Acid Antibiotics Thiolactomycin, Thiotetromycin, and 834-B1 Angew. Chem., Int. Ed. 461160 
17. Liao M, Peng L and Wang J $2008 \mathrm{Rh}(\mathrm{II})$-Catalyzed Sommelet-Hauser Rearrangement Org. Lett. 10693

18. (a) Reddy A C S, Choutipalli V S K, Ghorai J, Subramanian V and Anbarasan P 2017 Stereoselective Palladium-Catalyzed Synthesis of Indolines via Intramolecular Trapping of N-Ylides with Alkenes ACS Catal. 7 6283; (b) Yadagiri D and Anbarasan P 2015 Tandem 1, 2-sulfur migration and (aza)-DielsAlder reaction of $\beta$-thio- $\alpha$-diazoimines: rhodium catalyzed synthesis of (fused)-polyhydropyridines, and cyclohexenes Chem. Sci. 6 5847; (c) Yadagiri D, Reddy A C S and Anbarasan P 2016 Rhodium catalyzed diastereoselective synthesis of 2,2,3,3-tetrasubstituted indolines from N-sulfonyl-1,2,3-triazoles and ortho-vinylanilines Chem. Sci. 7 5934; (d) Yadagiri D and Anbarasan P 2013 Rhodium-catalyzed denitrogenative [2,3] sigmatropic rearrangement: an efficient entry to sulfur-containing quaternary centers Chem. Eur. J. 1915115

19. Muthusamy S, Gunanathan C, Babu S A, Suresh E and Dastidar P 2002 First example of regiospecific intermolecular $\mathrm{C}-\mathrm{H}$ insertion reactions of cyclic rhodium carbenoids: novel synthesis of 3-indol-3'-yloxindoles Chem. Commun. 824

20. Venkat Ragavan R, Vijayakumar V and Suchetha Kumari N 2009 Synthesis of some novel bioactive 4-oxy/thio substituted-1H-pyrazol-5(4H)-ones via efficient cross-Claisen condensation Eur. J. Med. Chem. 443852

21. Bhalla A, Sharma S, Bhasin K K and Bari S S 2007 Convenient Preparation of Benzylseleno- and Phenylselenoalkanoic Acids: Reagents for Synthesis of Organoselenium Compounds Synth. Commun. 37783

22. CCDC 1944163 